\title{
Cenários de desmatamento para a Amazônia
}

\author{
Britaldo Silveira SoARES-Filho, DANIEL C. NEPSTad, \\ Lisa CURRan, GUSTaVo CoUtinho CERQUEIRA, \\ RICARDO A. GARCIA, CLAUDIA AZEVEDO RAMOS, \\ ELIANE VOLL, ALICE MCDONALD, PAUL LEFEBVRE, \\ PETER SCHLESINGER e DAVID MCGRATH
}

\section{Causas do desmatamento na Amazônia e suas implicações futuras}

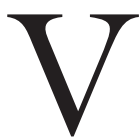

ASTAS MUDANÇAS estão em curso na Amazônia, como evidenciado pelo rápido avanço do desmatamento. Enquanto Achard et al. (2002) estimaram uma perda florestal de 150 mil km² para toda América Latina entre 1990 e 1997, nesse mesmo período, somente para a Amazônia brasileira, o projeto Prodes (Inpe, 2004) mensurou cerca de $100 \mathrm{mil} \mathrm{km}^{2}$ de perda florestal. Dados desse projeto demonstram ainda que as taxas brutas de desmatamento, que atingiram 23 mil km² ano-1 entre o biênio 2002-2003, vêm se acelerando através do último qüinqüênio em cerca de $13 \%$ ao ano.

As causas históricas e presentes do desmatamento na Amazônia são diversas e freqüentemente inter-relacionadas. Compreendem desde incentivos fiscais (Mahar, 1988; Schmink e Wood, 1992; Moran, 1993) e políticas de colonização no passado (Hecht, 1985; Hecht e Cockburn, 1990; Schmink e Wood, 1992; Andersen e Reis, 1997; Laurance, 1999), as quais desencadearam uma forte migração para Amazônia como válvula de escape para os problemas sociais de outras regiões (Skole et al., 1994); recorrentes conflitos fundiários motivados pela ausência de titularidade da terra e pela pressão da reforma agrária (Fearnside, 1985 e 2001; Soares-Filho et al., 2004); até o recente cenário macroeconômico (Margulis, 2002), envolvendo o avanço da exploração madeireira (Nepstad et al., 2001), da pecuária (Mertens et al., 2002; Kaimowitz et al., 2004) e o boom do agronegócio (Figura 1), notadamente a expansão das culturas de soja sobre áreas de pastagens (Alencar et al., 2004a). Investimentos em infra-estrutura, sobretudo a abertura de estradas e pavimentação (Nepstad et al., 2000; Carvalho et al., 2001; Laurance et al., 2001), completam esse quadro, posto que promovem a viabilidade econômica da agricultura e da exploração madeireira na Amazônia central, com conseqüente valorização de suas terras.

A previsão de asfaltamento de rodovias através da região (Nepstad et al., 2000 ) estimulará ainda mais a expansão da fronteira agrícola e da exploração ma- 
deireira, podendo acarretar uma colossal conversão de florestas em pastagens e áreas agrícolas, e, conseqüentemente, profunda perda do patrimônio genético de vários ecossistemas da Amazônia - ainda pouco conhecido -, e redução regional das chuvas (Silva Dias et al., 2002), com resultante aumento da flamabilidade de suas paisagens (Nepstad et al., 1999) e extensiva savanização (Nobre et al., 1996). Somam-se a isso as contribuições dessas mudanças para o aquecimento global, posto que o desmatamento representa hoje cerca de $75 \%$ das emissões de $\mathrm{CO}^{2}$ brasileiras (Houghton et al., 2000), e suas teleconexões climáticas - alterações no clima de outras regiões -, como a diminuição de chuvas no sudeste brasileiro (Fearnside, 2003) e o agravamento do período de estiagem no meiooeste americano (Avissar \& Werth, 2002).

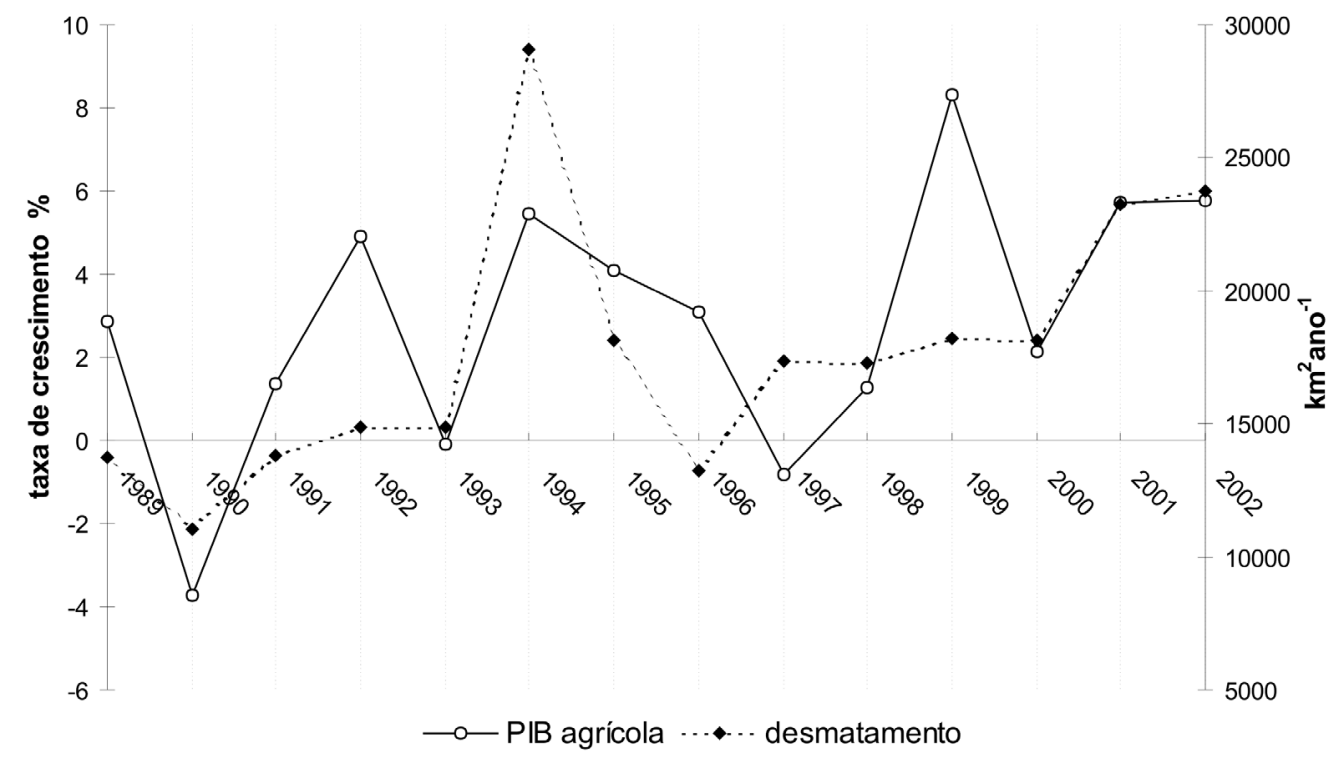

Figura 1 - Série temporal da taxa bruta anual de desmatamento (Inpe, 2004), comparada com a taxa de crescimento anual do PIB agrícola. Dados do Prodes (Inpe, 2004) são relativos aos biênios entre 1988-1989 a 2002-2003.

Por conseguinte, essas grandes mudanças na cobertura florestal têm importantes implicações quanto à perda de biodiversidade e outros serviços ambientais, emissão de gases que contribuem para o efeito estufa e à prosperidade da sociedade da Amazônia a longo prazo. Nessa perspectiva, um importante desafio para a comunidade científica consiste em simular os efeitos da infra-estrutura de transporte nos padrões regionais de mudanças de uso e cobertura do solo. A avaliação dos impactos indiretos dessas mudanças é de particular interesse tanto para planejadores regionais como para cientistas que estudam as mudanças climáticas. O desenho de uma estratégia de conservação para a floresta amazônica dependerá do rápido avanço na nossa compreensão das conexões da floresta com seus ecossistemas nativos e vida silvestre, clima regional, em conjunto com a economia e bem-estar da sociedade local. O projeto "Cenários para a Amazônia" 
(www.amazonscenarios.org), de caráter inter-institucional, busca desenvolver um modelo integrado, baseado em um ambiente computacional, que simule a dinâmica de uso e cobertura do solo na Amazônia, seus impactos nos ecossistemas amazônicos e as complexas interações entre esses ecossistemas com os climas regional e global, e ciclos hidrológicos das principais baciais hidrográficas. Além de possibilitar o estudo das retro-alimentações entre esses sistemas, o modelo integrado é também concebido como um instrumento de avaliação do potencial de políticas públicas para a conservação da Amazônia.

Dentro do escopo do referido projeto, neste artigo é descrito um modelo de simulação espacial de desmatamento na bacia Amazônica, sensível a diferentes cenários de políticas públicas frente à expansão da infra-estrutura de transporte na região. À luz do modelo, são analisados os impactos de uma gama de cenários, variantes da tendência histórica, na fragmentação florestal das paisagens da Amazônia. Os mapas de mudanças da cobertura florestal produzidos pelo modelo em questão encontram-se disponíveis para estudos de avaliação de seus impactos ambientais indiretos, como emissão de carbono para atmosfera, redução de hábitats, perda de biodiversidade, alteração do ciclo hidrológico na bacia e mudanças nos climas regional e global (www.csr.ufmg.br/simamazonia).

\section{Projetos de pavimentação de estradas na Amazônia}

A pavimentação e a construção de estradas consistem no principal determinante dos futuros padrões de desmatamento da bacia Amazônica. Atualmente, vários projetos de pavimentação estão sendo considerados pelo governo brasileiro: as obras de pavimentação de um trecho de cerca de $700 \mathrm{~km}$ da BR-163, da divisa do Pará com o Mato Grosso ao porto de Itaituba, estão marcadas para serem iniciadas em breve. Grandes interesses econômicos estão por trás desse projeto que visa a conectar a região produtora de soja do Mato Grosso a portos de calado internacional do sistema fluvial do Amazonas. Outros projetos de pavimentação incluem a BR-230 (rodovia Transamazônica), BR-319 (rodovia Manaus-Porto Velho), BR-156 do Amapá a Guiana Francesa, BR-401 de Roraima a Guiana, assim como muitos outros trechos de importância secundária (Figura 2).

Além disso, projetos de integração continental contemplam a pavimentação de rodovias através dos Andes, ligando não só a Amazônia mas o restante do Brasil a portos no Pacífico, como Callao no Peru e Arica no Chile. Dentre esses projetos, prioridade foi dada ao asfaltamento do trecho da rodovia Transamericana entre Assis Brasil, no Acre, a Cuzco, no Peru, a qual já se encontra asfaltada daí para o Pacífico. Como alternativa, vislumbra-se também uma ligação entre Cruzeiro do Sul, no Acre, a Pucalpa, no Peru. Existe, igualmente, a possibilidade de se construir uma rodovia ligando Cárceres, no Mato Grosso, a Santa Cruz na Bolívia. Santa Cruz, localizada no interior da bacia Amazônica, é hoje um centro urbano em franca expansão, com importância econômica maior do que a sua capital La Paz, graças aos seus campos de gás natural. Essa rota, embora até então não priorizada, representa a conexão mais curta entre as regiões industriais e al- 
tamente povoadas do sudeste brasileiro aos portos do norte do Chile, atravessando ainda a região produtora de soja do Brasil central. Por fim, outros projetos de transporte fluvial, construção de barragens, hidrelétricas e gasodutos completam esse quadro de investimentos em infra-estrutura para a Amazônia (Nepstad et al., 2000; Carvalho et al., 2001; Laurance et al., 2001).

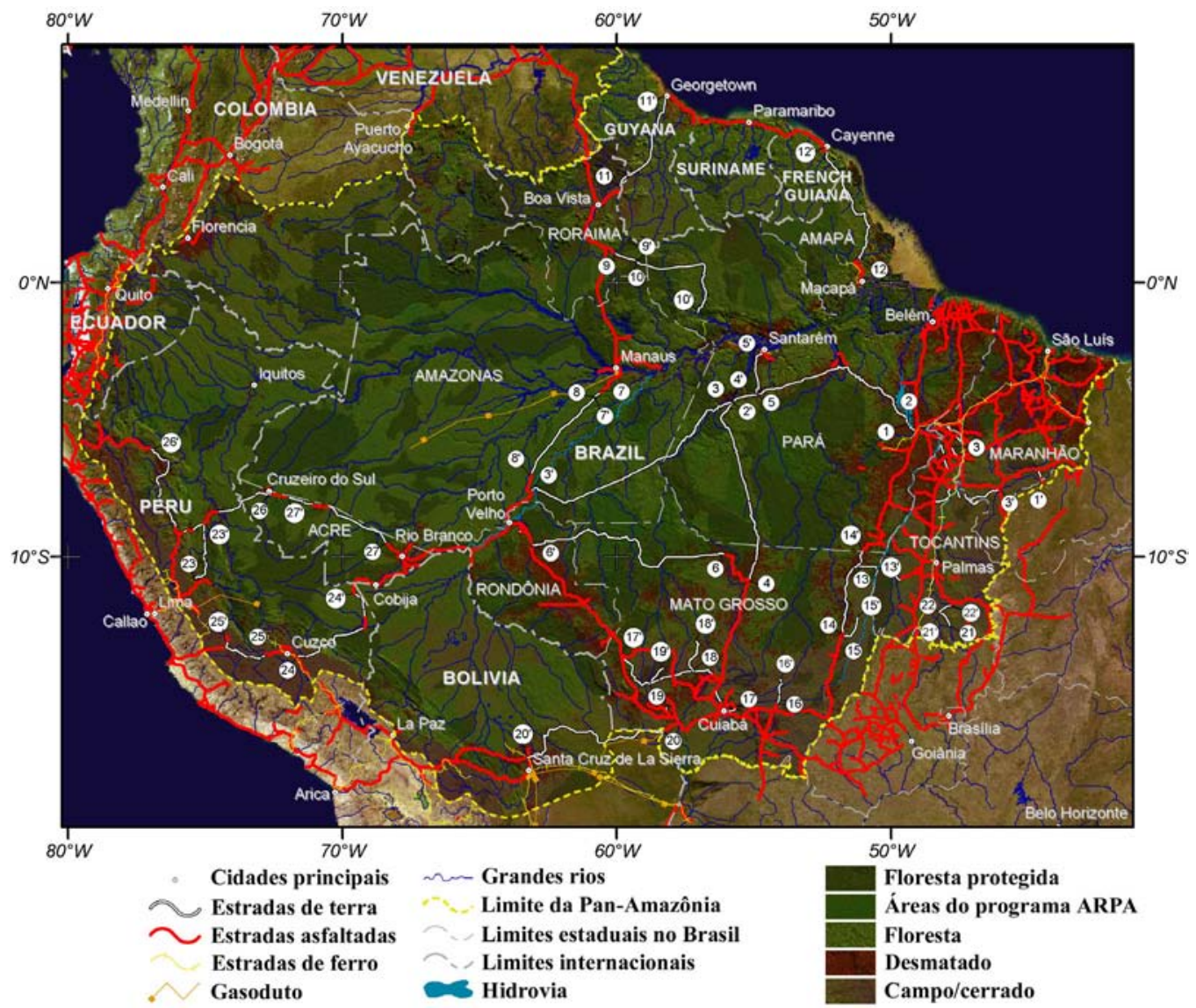

Figura 2 - Bacia Amazônica, principais cidades, estradas pavimentadas e de terra e áreas protegidas atuais e planejadas. Trechos rodoviários a serem asfaltados indicados por números indexados à Tabela 1.

O modelo em questão focaliza o efeito da pavimentação de rodovias na futura trajetória do desmatamento, haja vista que a cadeia de efeitos de outros investimentos em infra-estrutura ainda é bastante indeterminada. Naturalmente, o impacto do asfaltamento de estradas nas mudanças de cobertura do solo, nos movimentos migratórios e no bem-estar das sociedades que vivem nessas regiões dependerá da efetividade de esforços em conservação e ordenamento territorial levados a cabo atualmente. À luz das tendências atuais, investimentos em integração regional, sobretudo através da pavimentação de rodovias, devem ocorrer sob o espectro de um entre dois cenários plausíveis: "o mesmo de sempre" (businessas-usual), no qual as forças de destruição continuam sem efetiva contraposição, 
e um cenário de "governança", no qual os vários segmentos da sociedade, em conjunto com o Estado, desempenham um importante papel em prol da utilização regulada dos recursos naturais e conseqüente conservação da integridade ambiental da bacia amazônica (Nepstad, et al., 2002; Soares-Filho et al., 2004). Portanto, a prevalência de um desses cenários determinará o alcance do desmatamento através da bacia, à medida que novas infra-estruturas são criadas e os mercados nacional e internacional crescem, trazendo consigo viabilidade econômica para a agricultura e a exploração madeireira nas terras centrais da Amazônia.

Com o intuito de incorporar a influência da pavimentação de rodovias na projeção de desmatamento, foi estabelecido um calendário especificando para as três próximas décadas as datas prováveis de término do asfaltamento de cada trecho viário previsto, tendo como base documentos oficiais e conversas com agentes públicos (Tabela 1). Essas novas estradas asfaltadas exercerão um efeito no desmatamento, não só aumentando as suas taxas regionais, mas também iniciando novas fronteiras de ocupação.

\section{Desenho do modelo}

O ambiente de simulação desenvolvido incorpora dois modelos acoplados com distintas estruturas espaciais: 1) uma configuração em sub-regiões definida a partir de uma regionalização socioeconômica da Amazônia (Garcia et al., 2004) (Figura 3) e 2) um mapa raster (estrutura matricial) composto por 3144 x 4238 células a $1 \mathrm{~km}^{2}$ de resolução ${ }^{1}$. Um modelo integrador de cenários projeta as taxas de desmatamento para as 47 sub-regiões da bacia, processando dados de desmatamento (taxa anual e derivada média anual calculada para o qüinqüênio 19972002) $)^{2}$, de estradas a serem asfaltadas (Tabela l) e das extensões de remanescentes florestais e áreas protegidas atuais e planejadas em cada uma das sub-regiões.

As taxas regionais produzidas por esse modelo são então passadas para um simulador de mudanças espaciais - Dinamica (Soares-Filho et al., 2005). Esse modelo corresponde a um sistema de informação geográfica (SIG) de caráter dinâmico, o qual utiliza mapas de infra-estrutura (rodovias, estradas vicinais, estradas de ferro, gasodutos, canais fluviais e portos), unidades administrativas (estados, países e macrorregiões), áreas protegidas (unidades de conservação federal e estadual e terras indígenas), e aspectos biofísicos (vegetação, solo e topografia) para localizar as áreas mais prováveis a serem desmatadas (Soares-Filho et al., 2004) e assim reproduzir os padrões de progressão do desmatamento através do território amazônico. Cada sub-região possui um modelo singular com parâmetros personalizados. Não obstante, a integridade espacial entre as sub-regiões é assegurada pelo cômputo anual de um subconjunto de variáveis espaciais (e.g. distâncias ao desmatamento prévio e às estradas vicinais) de modo contínuo por toda a bacia. Isso faz com que a dinâmica de uma região afete as de suas vizinhas. Também, conectado ao simulador de mudanças encontra-se um modelo para simular a expansão da rede de estradas vicinais e assim incorporar o efeito da abertura de estradas espontâneas (Souza et al., 2004) na difusão do desmatamento. 
Tabela 1 - Calendário de asfaltamento de estradas

\begin{tabular}{|c|c|c|c|c|}
\hline chave & sigla & nome & trecho a ser pavimentado & $\begin{array}{c}\text { término da } \\
\text { pavimentação }\end{array}$ \\
\hline $1-1^{\prime}$ & BR-230 & Transamazônica & $\begin{array}{l}\text { de Araguatins (TO) para } \\
\text { Itupiranga (PA) }\end{array}$ & 2008 \\
\hline $2-2^{\prime}$ & BR-230 & Transamazônica & $\begin{array}{l}\text { de Itupiranga (PA) } \\
\text { para BR-163 }\end{array}$ & 2012 \\
\hline $3-3^{\prime}$ & BR-230 & Transamazônica & $\begin{array}{l}\text { de TO-040 para GO-118 e } \\
\text { trechos no MA e TO }\end{array}$ & 2025 \\
\hline $4-4^{\prime}$ & BR-163 & $\begin{array}{l}\text { Cuiabá- } \\
\text { Santarém }\end{array}$ & $\begin{array}{c}\text { da interseção de Colíder (MT) } \\
\text { para BR-230 } \\
\text { (Transamazônica) }\end{array}$ & 2008 \\
\hline $5-5^{\prime}$ & BR-163 & $\begin{array}{l}\text { Cuiabá- } \\
\text { Santarém }\end{array}$ & $\begin{array}{c}\text { de BR-230 (Transamazônica) } \\
\text { para Santarém }\end{array}$ & 2008 \\
\hline $6-6^{\prime}$ & & & $\begin{array}{c}\text { entre Alta Floresta (MT) e } \\
\text { BR-364, próximo a } \\
\text { Ariquemes (RO) }\end{array}$ & 2025 \\
\hline $7-7^{\prime}$ & BR-319 & $\begin{array}{c}\text { Manaus- } \\
\text { Porto Velho }\end{array}$ & $\begin{array}{c}\text { de } 160 \mathrm{~km} \text { ao sul da } \\
\text { BR-174 para sul }\end{array}$ & 2012 \\
\hline $8-8^{\prime}$ & BR-319 & $\begin{array}{c}\text { Manaus- } \\
\text { Porto Velho }\end{array}$ & $\begin{array}{c}\text { de } 195 \mathrm{~km} \text { ao sul da } \\
\text { BR-174 para sul }\end{array}$ & 2018 \\
\hline $9-9$ & BR-210 & & $\begin{array}{l}\text { do quilômetro } 75 \text { para } \\
\text { quilômetro } 175\end{array}$ & 2008 \\
\hline $10-10^{\prime}$ & BR-210 & & de quilômetro 175 & 2025 \\
\hline$\overline{11-11^{\prime}}$ & BR-401 & & $\begin{array}{l}\text { de Bomfim (RR) para } \\
\text { Queenstown (Guyana) }\end{array}$ & 2012 \\
\hline 12-12' & BR-156 & $\begin{array}{l}\text { Cayenne- } \\
\text { Oiapoque }\end{array}$ & $\begin{array}{c}\text { de } 150 \mathrm{~km} \text { ao norte de } \\
\text { Santana (AP) para Matoury } \\
\text { (French Guiana) }\end{array}$ & 2018 \\
\hline 13-13' & & & BR-158 para Vila Rica (MT) & 2008 \\
\hline 14-14' & BR-158 & & $\begin{array}{c}\text { entre } 220 \mathrm{~km} \text { ao norte de } \\
\text { Nova Xavantina (MT) e } \\
250 \mathrm{~km} \text { ao sul de } \\
\text { Redenção (PA) }\end{array}$ & 2008 \\
\hline $15-15^{\prime}$ & & & $\begin{array}{c}\text { este da BR-158, de } 220 \mathrm{~km} \\
\text { norte de Nova Xavantina (MT) }\end{array}$ & 2008 \\
\hline $16-16^{\prime}$ & $\mathrm{MT}-130$ & & $\begin{array}{l}\text { de } 23 \mathrm{~km} \text { norte de Primavera } \\
\text { do Leste (MT) para MT-110 }\end{array}$ & 2008 \\
\hline $17-17^{\prime}$ & BR-364 & & $\begin{array}{l}\text { de Chapada dos Guimarães } \\
\text { (MT) para BR-174; }\end{array}$ & 2012 \\
\hline $18-18^{\prime}$ & & & $\begin{array}{l}\text { de } 85 \mathrm{~km} \text { a este de } \\
\text { MT-170 para norte }\end{array}$ & 2012 \\
\hline $19-19^{\prime}$ & & & $\begin{array}{l}\text { de } 90 \mathrm{~km} \text { a oeste de } \\
\text { MT-170 para norte }\end{array}$ & 2018 \\
\hline $20-20^{\prime}$ & & $\begin{array}{c}\text { Cáceres- } \\
\text { Santa Cruz } \\
\end{array}$ & $\begin{array}{c}\text { entre Montero e San Matias } \\
\text { (Bolivia) }\end{array}$ & 2012 \\
\hline $21-21^{\prime}$ & GO-255 & & de Paranã (TO) para TO-280 & 2008 \\
\hline $22-22$ & TO-280 & & de GO-255 para TO-040 & 2008 \\
\hline $23-23^{\prime}$ & $5 \mathrm{~N}$ & & $\begin{array}{c}\text { de } 10 \mathrm{~km} \text { este de Vila Rica } \\
\text { para oeste, Tingo María } \\
\text { (Peru) para } 16 \mathrm{~N}\end{array}$ & 2018 \\
\hline $24-24^{\prime}$ & $26 \mathrm{~B}$ & Transamericana & $\begin{array}{c}\text { de Cuzco para Puerto } \\
\text { Maldonado (Peru) e de } 45 \mathrm{~km} \\
\text { norte de Puerto Maldonado } \\
\text { para Iberia (Peru) }\end{array}$ & 2008 \\
\hline $25-25^{\prime}$ & $3 \mathrm{~S}$ & & $\begin{array}{l}\text { de } 100 \mathrm{~km} \text { para } 300 \mathrm{~km} \text { ao } \\
\text { oeste de Cuzco (Peru) }\end{array}$ & 2008 \\
\hline $26-26^{\prime}$ & $\begin{array}{l}\text { BR-364, } \\
16 \mathrm{~B}, 5 \mathrm{~N}\end{array}$ & & $\begin{array}{c}\text { de Cruzeiro do Sul (AC) para } \\
\text { Mayobamba (Peru) }\end{array}$ & 2018 \\
\hline $27-27^{\prime}$ & BR-364 & & $\begin{array}{c}\text { de 90km norte de Senador } \\
\text { Guiomard (AC) para } \\
\text { Feijó (AC); }\end{array}$ & 2008 \\
\hline
\end{tabular}

TO (Tocantins); PA (Pará); GO (Goiás); MT (Mato Grosso); RO (Rondônia); RR (Roraima); AP (Amapá); AC (Acre). 


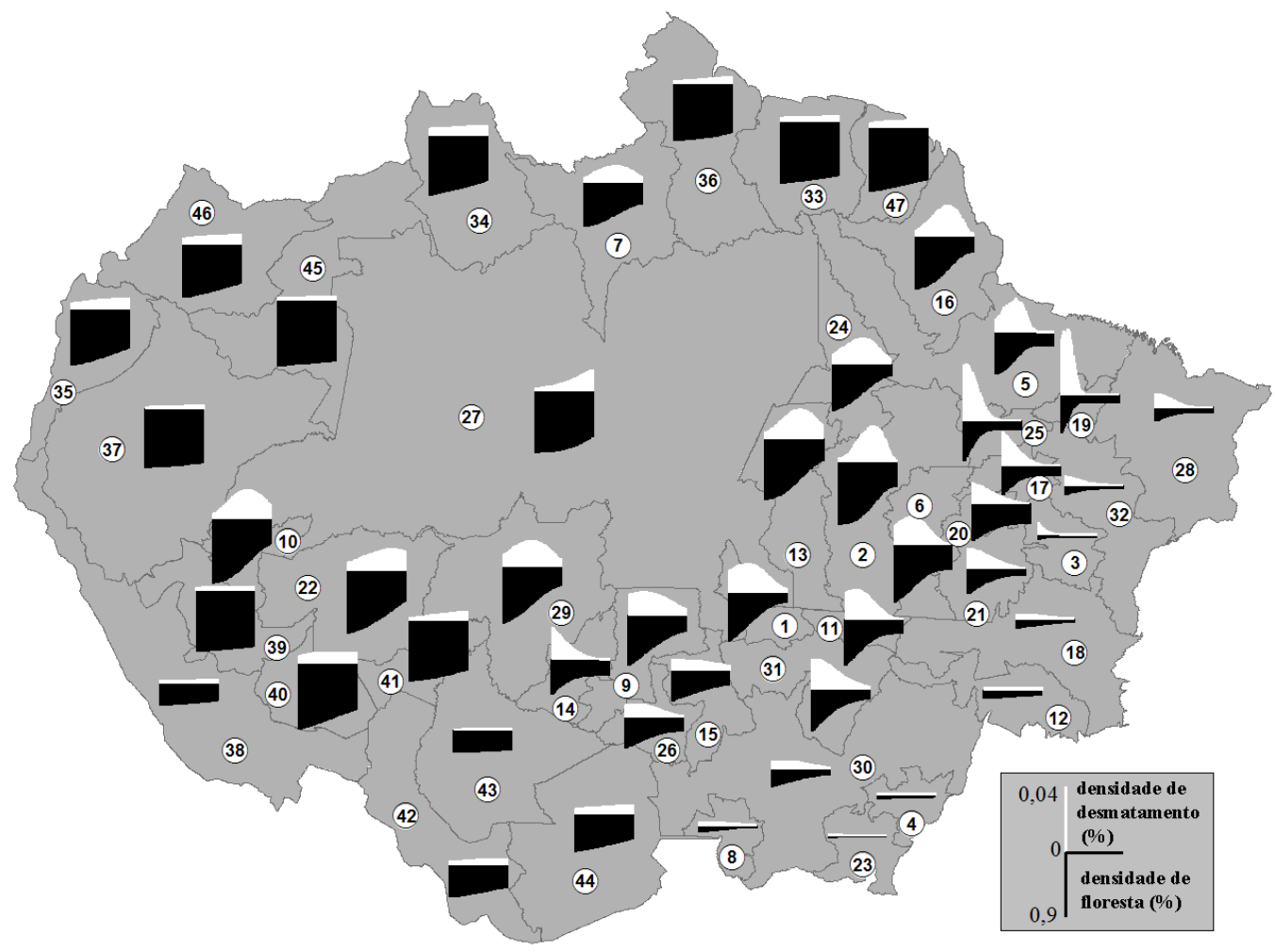

Figura 3 - Mapa de regionalização da bacia Amazônica, mostrando a projeção das taxas regionais de desmatamento e do declínio da floresta entre 2001 a 2050.

\section{Modelagem de cenários}

O modelo de simulação foi rodado para oito cenários, em passos anuais, por um intervalo de tempo de cinqüenta anos a partir de 2001. O cenário mais pessimista, denominado "o mesmo de sempre", considera as tendências históricas de desmatamento através da bacia, realizando as projeções pela multiplicação da taxa do ano anterior por sua derivada média anual entre 1997 e 2002 e adicionando a esse termo um fator de aceleração advindo da pavimentação de um conjunto de estradas. Em todos os cenários, a pavimentação de estradas segue o calendário predefinido (Tabela 1 ), sendo seu efeito na aceleração do desmatamento estimado empiricamente, comparando-se a relação histórica entre a proporção de área desmatada com a densidade média de estradas asfaltadas em municípios da Amazônia brasileira. O cenário otimista de governança também considera as tendências históricas de desmatamento através da bacia, mas, nesse caso particular, a projeção do desmatamento assume uma curva na forma de U invertido, reflexo do aumento gradual do estado de governança através da Amazônia. Nesse cenário, o desmatamento não ultrapassa $50 \%$ da cobertura florestal original em propriedades privadas, enquanto para o cenário "o mesmo de sempre" esse limite é estendido a $85 \%$. Observe-se que esses patamares são superiores ao estabelecido pelo código florestal brasileiro - único país amazônico a possuir lei com essa 
especificidade -, porém mais realistas. O cenário de governança assume que a rede de áreas protegidas será expandida, como proposto pelo Programa Arpa (Áreas Protegidas da Amazônia) (Montiel, 2004), sendo que nesse cenário é assegurada a proteção integral das áreas protegidas, enquanto que em "o mesmo de sempre”, essas áreas podem perder, a longo prazo, até $40 \%$ de sua cobertura florestal original por carência de fiscalização ambiental. Como resultado, a taxa de desmatamento inicialmente aumenta, devido à pressão advinda das novas estradas pavimentadas, até um certo limiar, dependendo dos limites preestabelecidos, quando passa a declinar em virtude da escassez de florestas remanescentes.

Seis cenários intermediários foram também analisados, alternando-se alguns pressupostos: 1) cenário de governança, mas sem novos asfaltamentos, 2) cenário de governança sem a inclusão das áreas do Arpa, 3) "o mesmo de sempre" com a inclusão das áreas do Arpa e ostensiva fiscalização ambiental a fim de se garantir a preservação integral dessas áreas 4) “o mesmo de sempre” sem áreas do Arpa, mas com ostensiva fiscalização ambiental das áreas protegidas atuais, 5) "o mesmo de sempre" com a inclusão de novas áreas do Arpa, mas sem ostensiva fiscalização ambiental, o que pode permitir até $40 \%$ de desmate dessas áreas, e 6 ) cenário histórico, compreendendo apenas a tendência recente de aceleração do desmatamento.

\section{Mudanças esperadas}

As projeções de desmatamento para os cenários extremos mostram trajetórias distintas. Sob o cenário "o mesmo de sempre", as taxas anuais ascendem dos patamares atuais de 23 mil e 28 mil km$^{2} \mathrm{ano}^{-1}$, respectivamente para o Brasil e para a bacia como um todo, para cerca de 40 mil a $48 \mathrm{mil} \mathrm{km}^{2} \mathrm{ano}^{-1}$, perto de 2030, declinando-se levemente daí em diante. Em contraste, no cenário de governança, as taxas assumem uma trajetória descendente graças ao aumento gradual do estado de governança através da bacia (Figura 4).

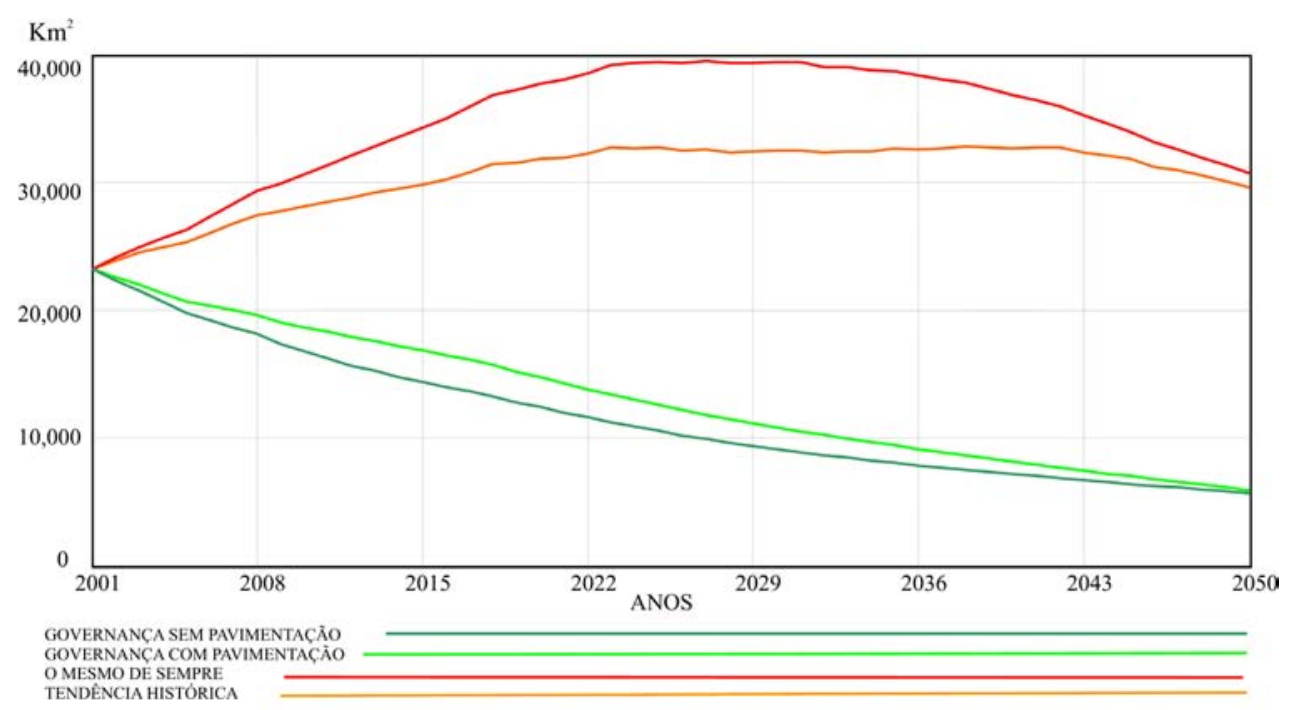

Figura 4 - Projeção do desmatamento sob vários cenários para a Amazônia brasileira. 
Como resultado, a tendência prevista de desmatamento dentro do cenário "o mesmo de sempre" levará, em meados deste século, a uma redução de cerca de $40 \%$ nos atuais 5,4 milhões de $\mathrm{km}^{2}$ de florestas da bacia Amazônia. Para a Amazônia brasileira, esses números são ainda mais assustadores, já que as perdas podem ultrapassar $50 \%$ de seus atuais 3,3 milhões $\mathrm{km}^{2}$ de florestas. Ao efeito da pavimentação de estradas, podem-se creditar, ao longo do tempo, aproximadamente 250 mil $\mathrm{km}^{2}$ de desmatamento sobre a já vertiginosa tendência histórica da bacia como um todo, ou seja, mais do que uma década de desmatamento brasileiro, considerando a sua taxa atual. Todavia, se consideramos uma projeção à taxa atual imutável, esse adicional avulta-se para $680 \mathrm{mil} \mathrm{km}^{2}$ até 2050, o que equivale ao total atual da área desmatada na Amazônia brasileira ${ }^{3}$.

Contextualizando esses números, demonstra-se que o leste e o sudeste amazônico serão as áreas mais atingidas. Em regiões como o leste do Pará e por todo o estado do Mato Grosso, grandes extensões de florestas fora das áreas protegidas praticamente desaparecerão; mesmo unidades de conservação próximas às principais rodovias serão afetadas em grande extensão (Figura 5). Também as reservas indígenas, hoje consideradas mais bem protegidas (Nepstad et al., no prelo), terão seus ecossistemas nativos profundamente afetados, visto que estarão mais sujeitos ao fogo e secas prolongadas, resultantes do avanço do clima de savana sobre a bacia - especialmente as florestas de transição no parque do Xingu.

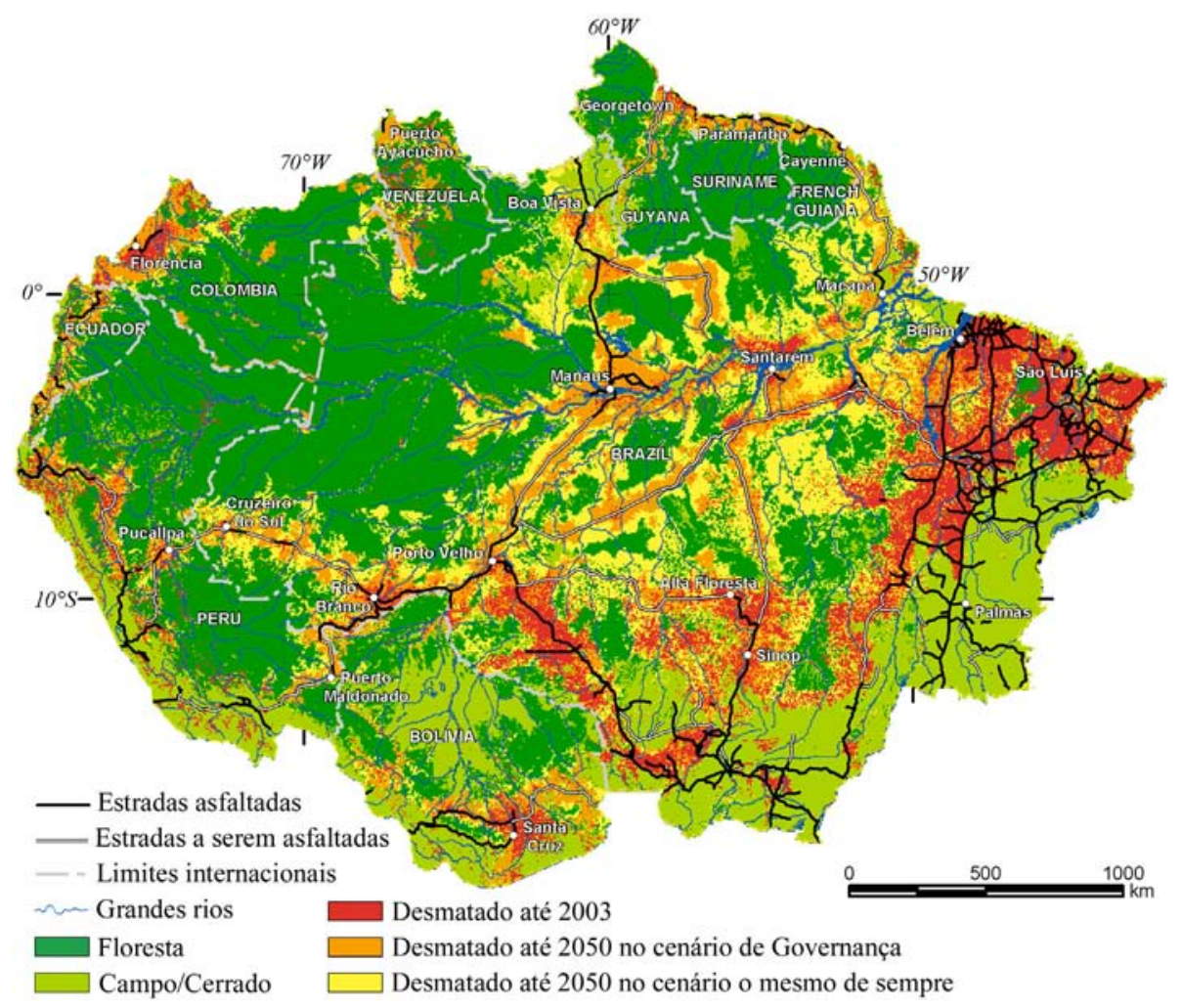

Figura 5 - Padrões espaciais de desmatamento simulados para os dois cenários extremos: governança x "o mesmo de sempre". 
Com efeito, por toda a bacia, novos corredores de desmatamento tenderão a se entrecruzar, gerando vastas conversões da cobertura florestal em regiões como o entorno de Manaus e eixos que se radiam daí em direção a Rondônia pela rodovia Manaus - Porto Velho, para norte por Roraima e a leste, ao largo do rio Amazonas. Em adição, serão afetadas largas faixas laterais às rodovias Transamericana e BR-364 Acre adentro, assim como amplas extensões de terra ao redor de Santa Cruz, na Bolívia, Florência, na Colômbia, e Puerto Ayacucho na Venezuela. Nesse cenário de fragmentação, somente regiões remotas, como o extremo noroeste da Amazônia brasileira e interior das Guianas ainda manterão grandes blocos coesos de floresta. Contudo, esta análise é ainda conservadora, pois não considera perturbações adicionais na floresta por fogo e exploração madeireira, nem outros projetos de estradas ainda não vislumbrados.

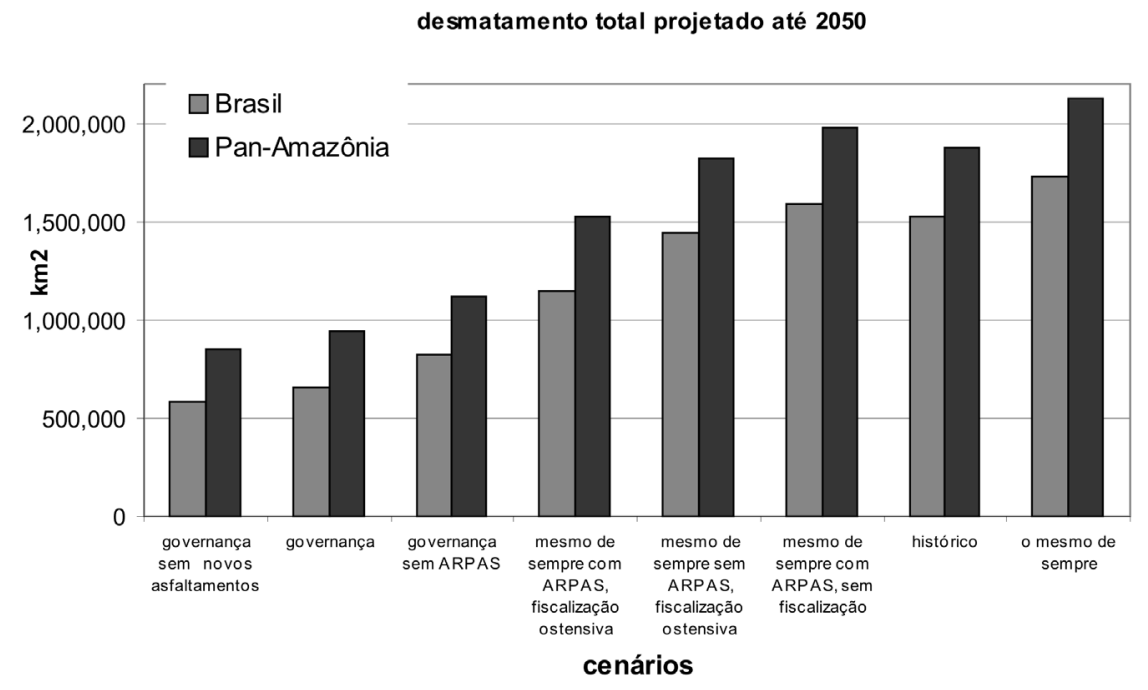

desmatamento reduzido em relação ao "mesmo de sempre" até 2050

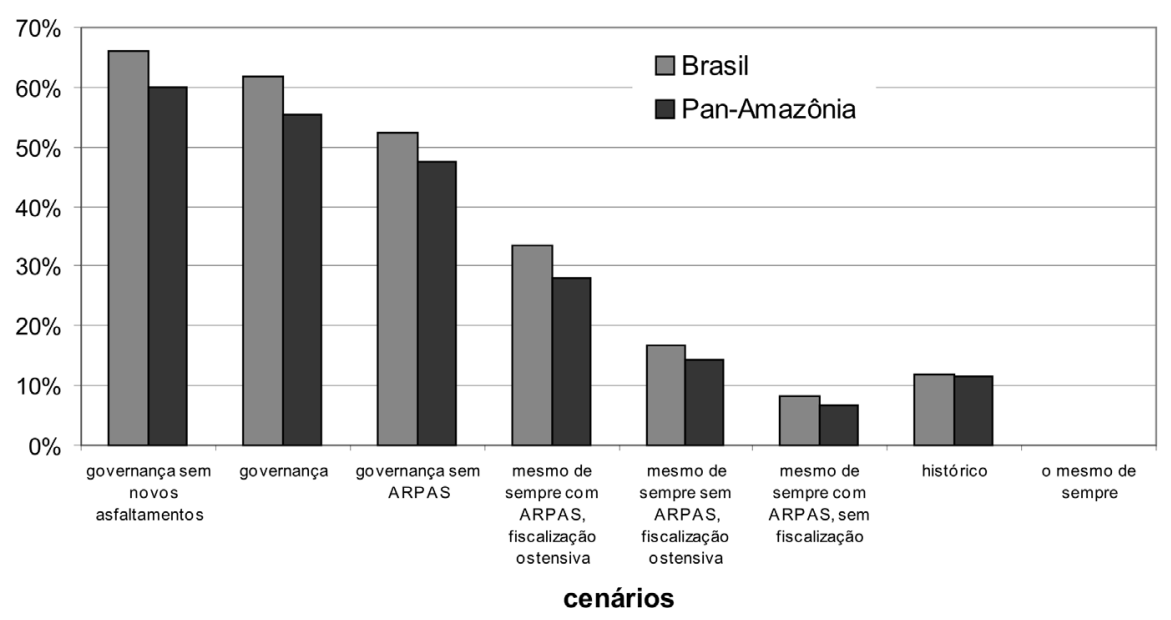

Figura 6 - Projeção do desmatamento total até 2050 em vários cenários e sua expectativa de redução tendo como base o cenário "o mesmo de sempre". 
Em contraste, um cenário de governança poderia reduzir o desmatamento previsto em até $62 \%$ e $55 \%$, respectivamente, para a Amazônia brasileira e a bacia como um todo, mesmo que se completassem todos os projetos planejados de asfaltamento (Figura 2). Seu resultado se expressa, portanto, pela expansão e preservação completa das áreas protegidas, aliadas à manutenção de um arranjo de paisagens rurais ecologicamente sustentáveis. Nesse aspecto, os cenários intermediários servem para analisar o papel das áreas protegidas na conservação da Amazônia. Tomando como base de comparação o desmatamento dentro do cenário "o mesmo de sempre", demonstra-se que somente a expansão da rede de áreas protegidas, de $29 \%$ (atualmente, $34 \%$ ) para $41 \%$ da Amazônia brasileira, mas sem sua implementação de fato, é capaz de reduzir o desmatamento previsto para o final da metade desta década em somente cerca de 7\% (Figura 6). Por outro lado, uma ostensiva fiscalização ambiental nessas áreas elevaria esse percentual para cerca de $30 \%$. Todas as medidas de conservação combinadas, mas sem a expansão das áreas protegidas, garantiriam $86 \%$ do desmatamento evitado pelo cenário de governança. Por fim, uma ampliada rede de áreas protegidas, efetivamente implementada via ostensiva fiscalização, seria responsável pela metade da redução no desmatamento atribuído ao cenário de governança, ou seja, pelo abatimento de um terço nas perdas florestais projetadas dentro do cenário "o mesmo de sempre".

\section{Estratégias de conservação}

Desenvolvimento na Amazônia sempre dividiu opiniões. Os resultados do modelo demostram que, de fato, a pavimentação de rodovias através do coração da Amazônia desencadeará uma vasta remoção de suas florestas, sobretudo se a tendência atual não for revertida a tempo. Observe-se que somente a expectativa de se asfaltar a BR-163 Pará adentro tem instigado a grilagem de glebas públicas, espalhado violência e, conseqüentemente, acelerado o desmatamento nessa região.

As conseqüências ambientais dessas mudanças são dramáticas. Dentro do cenário de "o mesmo de sempre", é esperado que mais de $2 / 3$ da cobertura vegetal de quinze principais ecorregiões amazônicas, de um total de trinta e duas, sejam eliminados, liberando aproximadamente $32 \operatorname{Pg}\left(10^{9}\right.$ toneladas $)$ de carbono para a atmosfera, o equivalente a mais de quatro anos das atuais emissões por todo o planeta. Grande extinção de espécies, muitas ainda não conhecidas, pode ocorrer na Amazônia oriental, onde as taxas de desmatamento são vertiginosas. A título de ilustração, 22\% de um total de 164 mamíferos analisados perderiam mais do que $40 \%$ de seu hábitat dentro da bacia (Soares-Filho et al., submetido).

Um cenário de ampla governança poderia reverter essa tendência, porém conciliar desenvolvimento com conservação não é trivial. Nos últimos anos, investimentos governamentais no controle do desmatamento têm aumentado, incluindo o crescimento do contingente do Ibama e o desenvolvimento de sistemas de detecção de desmatamento em tempo real - Deter (Inpe, 2005). Mas nem sempre essas medidas se traduzem em controle imediato, haja vista que o desmatamento segue em passo acelerado, estimulado pelo suposto progresso econômico da região. 
Unidades de conservação que garantam a preservação integral dos recursos naturais (parques nacionais e estaduais, estações ecológicas, reservas biológicas, entre outras) e áreas protegidas que permitam o uso desses recursos (terras indígenas, reservas extrativistas, reservas de desenvolvimento sustentável e florestas nacionais) são também componentes importantes da estratégia de controle do desmatamento. No entanto, os dados do modelo demonstram que mesmo um maciço investimento na implementação e manutenção de uma ampla rede de áreas protegidas, dentro de um cenário como "o mesmo de sempre", não seria suficiente para impedir o empobrecimento em larga escala das principais bacias hidrográficas, ecorregiões e hábitats amazônicos. Portanto, uma estratégia de conservação extensiva deve também envolver a proteção de um arranjo funcional de remanescentes florestais fora das áreas protegidas a fim de se evitar o colapso ambiental dos ecossistemas de florestas úmidas, já em curso em outras partes dos trópicos (Curran et al., 2004).

Experiências recentes em planejamento regional (Alencar et al., 2004b), zoneamento agro-ecológico (Sectma, 2000) e fiscalização ambiental (Fema, 2002) devem ser refinadas e multiplicadas para que consigamos sobrepujar as crescentes forças de explotação da floresta. Mas somente a presença da lei não basta, pois há, igualmente, necessidade de se valorizar a floresta em pé, buscando-se economias florestais, calcadas em uma sólida base macroeconômica, que sejam competitivas em face dos usos atuais em áreas convertidas, como a criação de gado e a plantação de grãos. Adiciona-se a essa estratégia a certificação ambiental para produtos de agricultores e fazendeiros que preservam a floresta em suas propriedades. Parte dos recursos necessários a esse esforço de conservação poderia vir na forma de créditos trocados por emissões de carbono evitadas, dentro de uma convenção do clima modificada, como discutido em recentes negociações (Santilli et al., no prelo). Observe-se que os $17 \mathrm{Pg}$ de emissão de carbono (16 Pg para o Brasil), evitados pelo cenário de governança em relação ao "mesmo de sempre", representam mais que oito vezes a redução nas emissões de gases causadores de efeito estufa a ser alcançada dentro do primeiro período de compensação do Protocolo de Kyoto. Além disso, vislumbram-se investimentos em cadeias de biotecnologia que explorem as enormes possibilidades do celeiro de biodiversidade amazônico. Enfim, essas medidas não somente trarão o bemestar para toda a sociedade amazônica, mas também a garantia de conservação desse primordial patrimônio natural da humanidade.

\section{Notas}

l O mapa de cobertura do solo para toda a bacia é formado por uma composição das cartas do Prodes de 2001 (Inpe, 2004), do mapa de vegetação da América do Sul (Eva et al., 2004) e da carta de desmatamento da Bolívia produzida por Steininger et al., 2001.

2 Os dados das séries do Prodes entre 1997 e 2002 (Inpe, 2004) foram empregados no cálculo das taxas de desmatamento das sub-regiões brasileiras. Para outras sub-regiões, 
com ausência de dados multitemporais, foram assinaladas taxas tomando como comparação sub-regiões brasileiras com tipologia e idade de fronteira similares.

3 Em vez de números absolutos, os resultados do modelo devem ser vistos como patamares a serem alcançados ao longo do curso de um dos cenários modelados.

\section{Referências}

ACHARD, F.; EVA, H. D.; STIBIG, H.; MAYAUX, P.; GALLEGO, J.; RICHARDS, T. e MALINGREAU, J. P. "Determination of Deforestation Rates of the World's Humid Tropical Forests”. Science, n. 297, 2002, pp. 999-1002.

ALENCAR, A. et. al. O desenvolvimento que queremos: ordenamento territorial da BR163, Baixo Amazonas, Transamazônica e Xingu. Meeting report. 29-31 mar., (IPAM, ISA, FVPP, Fetagri BAM, FORMAD, FVPP, CEFTBAM, Forum BR-163, GTA), Santarém, PA, 2004a.

ALENCAR, A.; NEPSTAD, D.; MCGRATH, D.; MOUTINHO, P.; PACHECO, P.; DEL CARMEN, M.; DIAZ, V. e SOARES FILHO, B. S. "Desmatamento na Amazônia: indo além da "emergência crônica". Ipam, 2004b http://www.ipam.org.br/ publicacoes/livros/resumo_desmatamento.php

ANDERSEN, L. E. e REIS, E. J. Deforestation, Development and Government Policy in the Brazilian Amazon: An Econometric Analysis. Working Paper 513, Rio de Janeiro, Instituto de Pesquisa Econômica Aplicada (Ipea), 1997.

AVISSAR, R. e WERTH, D. "Teleconnections between Tropical Deforestation and Midlatitude Precipitation". Eos. Trans. AGU. vol. 84, n. 46, Fall Meet. Suppl., Abstract, 2003. http://www.agu.org/meetings/fm03/fm03-pdf/fm03-B32A.pdf

CARVAlHO, G.; BARROS, A. C.; MOUTINHO, P. e NEPSTAD, D. "Sensitive Development Could Protect Amazonia Instead of Destroying It". Nature, n. 409, 2001, p. 131.

CURRAN, L. M. et al. "Lowland Forest Loss in Protected Areas of Indonesian Borneo". Science, n. 303, 2004, pp.1000-1003.

EVA, H. D.; BELWARD, A. S.; MIRANDA, E. E.; BELLA, C. M.; GOND, V.; HUBER, O.; JONES, S.; SGRENZAROLI, M. e FRITZ, S. “A Land Cover Map of South America”. Global Change Biology, vol. 10, n. 5, 2004, pp. 731-744.

FEARNSIDE, P. M. A Floresta Amazônica nas mudanças globais. Manaus, Instituto Nacional de Pesquisas da Amazônia (Inpa), 2003, 134 p.

“Agriculture in Amazonia”. Em PRANCE, G. T. e LOVEJOY, T. E. (eds.). Key Environments of Amazonia. Oxford, Pergamon Press, 1985, pp. 393-418.

"Land-tenure Issues as Factors in Environmental Destruction in Brazilian Amazonia: The Case of Southern Pará". World Dev. vol. 29, n. 8, 2001, pp.13611372 .

"Deforestation in Brazilian Amazonia: The Effect of Population and Land Tenure". Ambio, n. 22, 1993, pp. 537-545.

FEMA. Relatório dos resultados alcançados na implementação do sistema de controle ambiental de propriedades rurais no Estado do Mato Grosso. Cuiabá, Fundação Estadual do Meio Ambiente (Fema), Governo do Estado do Mato Grosso, 2002. 
GARCIA, R. A., SOARES-FILHO, B. S. e SAWYER, D. O. “Dimensões sócio-econômicas e movimentos populacionais: uma regionalização da Amazônia brasileira”. Em XIV Encontro Nacional de Estudos Populacionais. Caxambu, Abep, 2004. http:// www.abep.org.br/usuario/GerenciaNavegacao.php?caderno_id=030\&nivel=1

HECHT, S. "Environment, Development and Politics: Capital Accumulation and Livestock Sector in Eastern Amazonia”. Word Development, n. 13, 1985, pp. 663-684.

HECHT, S. e COCKBURN, A. The Fate of the Forest. Developers, Destroyers and Defenders of the Amazon. HarperCollins, 1990, 357 p.

HOUGHTON, R. A. et al. "Annual Fluxes of Carbon from Deforestation and Regrowth in the Brazilian Amazon". Nature, n .403, 2000, pp. 301-304.

INPE - Instituto Nacional de Pesquisas Espaciais. "Monitoramento da Floresta Amazônica Brasileira por Satélite - Projeto Prodes”. 2004: http://www.obt.inpe.br/prodes.

. Sistema Deter (Detecção de Desmatamento em Tempo Real). 2005. http:// www.obt.inpe.br/deter/

KAIMOWITZ, D.; MERTENS, B.; WUNDER, S. e PACHECO, P. "Hamburger Connection Fuels Amazon Destruction". Bangor, Indonesia, Center for International Forest Research, 2004. http://www.cifor.cgiar.org/publications/pdf_files/media/ Amazon.pdf

LAURANCE, W. F. "Reflections on Tropical Deforestation crisis". Biological Conservation, n. 91, 1999, pp. 109-117.

LAURANCE, W. F.; COCHRANE, M. A.; BERGEN, S.; FEARNSIDE, P. M.; DELAMÔNICA, P.; BARBER, C.; D’ANGELO, S. e FERNANDES, T. “The Future of The Brazilian Amazon". Science, n. 291, 2001, pp. 438-439.

MAHAR, D. J. Deforestation in Brazil's Amazon Region: Magnitude, Rate and Causes. New York, The World Bank, 1988.

MARGULIS, S. “Quem são os agentes dos desmatamentos na Amazônia e por que eles desmatam?" Word Bank internal paper. 2002. http://www.obancomundial.org/ index.php/content/view_folder/87.html]

MERTENS, B.; POCCARD-CHAPUIS, R.; PIKETTY, M.-G.; LACQUES, A.-E. e VENTURIERI, A. "Crossing Spatial Analyses and Livestock Economics to Understand Deforestation Processes in the Brazilian Amazon: The Case of São Félix do Xingu in South Pará”. Agricultural Economics, n. 27, 2002, pp. 269-294.

MONTIEL, F. Programa áreas protegidas da Amazônia arpa. Resumo Executivo, 2004. http://www.mma.gov.br/port/sca/ppg7/doc/arpareex.pdf

MORAN, E. F. "Deforestation and Land Use in the Brazilian amazon". Human Ecology, n. 21, 1993, pp. 1-21.

NEPSTAD, D. et al. "Inhibition of Amazon Deforestation and Fire by Parks and Indigenous Reserves". Conservation Biology, no prelo.

NEPSTAD, D.; CAPOBIANCO, J. P.; BARROS, A. C.; CARVALHO, G.; MOUTINHO, P.; LOPES, U. e LEFEBVRE, P. “Avança Brasil, The Environmental Costs for Amazônia". 2000. http://www.ipam.org.br/avanca/participen.htm

NEPSTAD, D.; MCGRATH, D.; ALENCAR, A.; BARROS, A. C.; CARVALHO, G.; SANTILLI, M. e VERA DIAZ, M. "Frontier Governance in Amazonia”. Science, n. 295, 2002, pp. 629-631. 
NEPSTAD, D.; VERISSIMO, A.; ALENCAR, A.; NOBRE, C.; LIMA, E.; LEFEBVRE, P.; SCHLESINGER. P.; POTTER, C.; MOUTINHO, P.; MENDOZA, E.; COCHRANE, M. e BROOKS, V. "Large-scale Impoverishment of Amazonian Forests by Logging and Fire”. Nature, n.398, 1999, pp. 505-508.

NEPSTAD, D.; CARVALHO, G.; BARROS, A. C.; ALENCAR, A.; CAPOBIANCO, J.; BISHOP, J.; MOUTINHO, P.; LEFEBVRE, P. e SILVA, U. "Road Paving, Fire Regime Feedbacks, and the Future of Amazon Forests". Forest Ecology and Management, n. 5524, 2001, pp.1-13.

NOBRE, C. A.; GASH, J. H. C.; ROBERTS, J. M.; VICTORIA, R. L. "Conclusions from Abraços”. Em GASH, J. H. C.; NOBRE, C. A.; ROBERTS, J. R. e VICTORIA, R. L. (eds.). "Amazonian Deforestation and Climate". Chichester, John Wiley and Sons, 1996.

SANTILLI, M. P. et al. "Tropical Deforestation and the Kyoto Protocol: An Editorial Essay". Climatic Change, no prelo.

SCHMINK, M. e WOOD, C. H. "Contested Frontiers in Amazonia”. New York, Columbia University Press, 1992, 385 p.

SECTMA. GOVERNO DO ESTADO DO ACRE. Programa Estadual de Zoneamento Ecológico-Econômico do Estado do Acre. Zoneamento ecológico-econômico: recursos naturais e meio ambiente - documento final. Rio Branco, vol. 1, 2000, 116 p.

SILVA DIAS, M. A. F. et al. "Cloud and Rain Processes in Biosphere-Atmosphere Interaction Context in the Amazon Region”. Journal of Geophysical Research, n. 107, 2002 , p. 8072.

SKOLE, D. L.; CHOMENTOWSKI, W. H.; SALAS, W. A. e NOBRE, A. D. "Physical and Human Dimensions of Deforestation in Amazonia". BioScience, vol. 44, n. 5, 1994, pp. 314-322.

SOARES FILHO, B.; ALENCAR, A.; NEPSTAD, D.; CERQUEIRA, G. C.; VERA DIAZ, M.; RIVERO, S.; SOLÓRZANO, L. e VOLL, E. "Simulating the Response of Land-Cover Changes to Road Paving and Governance Along a Major Amazon Highway: The Santarém-Cuiabá Corridor”. Global Change Biology, vol. 10, n. 7, 2004, pp. 745-764.

SOARES FILHO, B.; NEPSTAD, D.; CURRAN, L. e CERQUEIRA, G. C., “Conservation Scenarios for the Amazon". Nature, submetido.

SOARES FILHO, B. S.; RODRIGUES, H.; NEPSTAD, D.; VOLL, E. e ALENCAR, A. "Analysis of Spatial Determinants of Deforestation in Amazônia". EarthInteraction, submetido.

SOARES FILHO, B. S. e CERQUEIRA, G. C. Projeto DINAMICA. 2005. http:// www.csr.ufmg.br/dinamica

SOUZA, C.; BRANDÃO, A.; ANDERSON A. e VERÍSSIMO, A. "Avanço das estradas endógenas na Amazônia. Amazônia em Foco”. Manaus, Imazon, 2004. http:// www.imazon.org.br/upload/124466.pdf

STEININGER, M. K.; TUCKER, C. J.; TOWNSHEND, J. R. G.; KILLEEN, T. J.; DESCH, A.; BELL, V. e ERSTS, P. “Tropical Deforestation in the Bolivian Amazon”. Environmental Conservation, vol. 28, n. 2, 2001, pp. 127-134. 
RESUMO - A AMAZÔNIA está entrando em uma era de rápidas mudanças impulsionadas pela previsão de asfaltamento de rodovias que estimularão a expansão da fronteira agrícola e de exploração madeireira. O declínio do custo de transporte tem importantes implicações para a biodiversidade, emissão de gases que contribuem para o efeito estufa e prosperidade da sociedade da Amazônia a longo prazo. Para analisar esse contexto, foi desenvolvido um modelo de simulação de desmatamento na bacia Amazônica, sensível a diferentes cenários de políticas públicas frente à expansão da infra-estrutura de transporte pela região. Resultados do modelo indicam que, dentro de um cenário pessimista, o desmatamento projetado pode eliminar, até meados deste século, $40 \%$ dos atuais 5,4 milhões de $\mathrm{km}^{2}$ de florestas da Amazônia, liberando o equivalente a $32 \mathrm{Pg}$ ( $10^{9}$ toneladas) de carbono para atmosfera. A modelagem de cenários alternativos aponta que a expansão de uma rede de áreas protegidas, efetivamente implementadas, poderia reduzir em até $1 / 3$ as perdas florestais projetadas. Contudo, outras medidas de conservação são ainda necessárias para se manter a integridade funcional das paisagens e bacias hidrográficas amazônicas. Atuais experimentos em conservação florestal em propriedades privadas, mercados de serviços ambientais e zoneamento agro-ecológico devem ser refinados e multiplicados a fim de se buscar uma conservação extensiva.

Palavras-chave: Modelagem; simulação; cenários; desmatamento; Amazônia.

ABSRACT - THE AMAZON is entering an era of rapid changes as new transportation corridors traverse the region, stimulating the expansion of logging and agricultural frontiers. The declining cost of transportation has important implications for biodiversity, greenhouse gas emissions, and the long-term prosperity of the Amazon society. To analyze this context, we have developed an empirically based, policy-sensitive model of deforestation for the Amazon basin. Model output for the worst-case scenario shows that, by 2050 , projected deforestation trends will eliminate $40 \%$ of the current 5.4 million $\mathrm{km}^{2}$ of Amazon forests, releasing approximately $32 \mathrm{Pg}$ ( $10^{9}$ tons $)$ of carbon to the atmosphere. Results from intermediate-case scenarios indicate that, although an expanded and enforced network of protected areas could avoid as much as one third of projected forest losses, other conservation measures are still required to maintain the functional integrity of Amazon landscapes and watersheds. Current experiments in forest conservation on private properties, markets for ecosystem services, and agro-ecological zoning must be refined and implemented to achieve comprehensive conservation.

Key-words: Scenarios; simulation; modeling; deforestation; Amazon.

Britaldo Silveira Soares-Filho ${ }^{1,2}$, Daniel Curtis Nepstad ${ }^{3,5,6}$, Lisa Curran ${ }^{4}$, Gustavo Coutinho Cerqueira ${ }^{1}$, Ricardo Alexandrino Garcia ${ }^{2}$, Claudia Azevedo Ramos ${ }^{5}$, Eliane Voll $^{1}$, Alice McDonald ${ }^{4}$, Paul Lefebvre ${ }^{3}$, Peter Schlesinger ${ }^{3}$ e David McGrath ${ }^{3,5,6}$.

${ }^{1}$ Centro de Sensoriamento Remoto $/{ }^{2}$ Centro de Desenvolvimento e Planejamento Regional (Universidade Federal de Minas Gerais). @ - britaldo@csr.ufmg.br, cerca@csr.ufmg.br, voll@csr.ufmg.br e rica@cedeplar.ufmg.br; ${ }^{3}$ The Woods Hole Research Center (EUA). @ dnepstad@whrc.org, paul@whrc.org, pschles@whrc.org e dmcgrath@amazon.com.br; ${ }^{4}$ Tropical Resources Institute. Yale School of Forestry \&Environmental Studies (EUA); @ lisa.curran@yale.edu $e$ alice.mcdonald@yale.edu; ${ }^{5}$ Instituto de Pesquisa Ambiental da Amazônia (Belém, Pará).www.ipam.org.br@ - c.azevedoramos@terra.com.br; ${ }^{6}$ Universidade Federal do Pará, Núcleo de Altos Estudos Amazônicos, Campus do Guamá, Belém, Pará.

Texto recebido em 4/2/2005 e aceito em 18/4/2005.

Agradecem ao apoio do Projeto LBA, Fundação Gordon e Betty Moore e Capes. 